Proceedings

\title{
Numerical Investigations of Infrared Slot Waveguides for Gas Sensing ${ }^{\dagger}$
}

\author{
Christian Ranacher ${ }^{1, *}$ Andreas Tortschanoff ${ }^{1}$, Cristina Consani ${ }^{1}$, Reyhaneh Jannesari ${ }^{2}$, \\ Thomas Grille ${ }^{3}$ and Bernhard Jakoby ${ }^{2}$ \\ 1 Carinthian Tech Research AG, 9524 Villach, Austria; andreas.tortschanoff@ctr.at (A.T.); \\ cristina.consani@ctr.at (C.C.) \\ 2 Institute for Microelectronics and Microsensors, Johannes Kepler University, 4040 Linz, Austria; \\ reyhaneh.jannesari@jku.at (R.J.); bernhard.jakoby@jku.at (B.J.) \\ 3 Infineon Technologies Austria AG, 9500 Villach, Austria; thomas.grille@infineon.com \\ * Correspondence: christian.ranacher@ctr.at; Tel.: +43-4242-56300-237 \\ + Presented at the Eurosensors 2018 Conference, Graz, Austria, 9-12 September 2018.
}

Published: 30 November 2018

\begin{abstract}
Sensing of gases is a promising area for applications of photonic sensor devices that operate in the mid-infrared spectral range. We present a numerical investigation of slot waveguides for evanescent field sensing of $\mathrm{CO}_{2}$. The sensor platform is a poly-silicon slot waveguide on silicon dioxide, where both layers are deposited on a standard silicon substrate. The evanescent field ratio, which is a crucial parameter for the sensing performance of the waveguides, was determined and values as high as $42 \%$ were obtained.
\end{abstract}

Keywords: silicon photonics; mid-infrared sensing; slot waveguide

\section{Introduction}

In this work we present a numerical investigation of slot waveguides for evanescent field infrared absorption sensing of $\mathrm{CO}_{2}$. Slot waveguides were first theoretically described in [1], and experimentally demonstrated in [2]. Slot waveguides offer the advantage of an increased electric field in the slot that leads to a high evanescent field ratio, which is specifically beneficial for gas sensing applications. If $\mathrm{CO}_{2}$ is present in the slot, the evanescent field is partially absorbed by the gas, which is detected in terms of an associated damping of the guided wave. In [3], a numerical investigation of slot waveguides for $\mathrm{CO}_{2}$ sensing using a silicon on sapphire platform was presented and an evanescent field ratio as high as $\sim 25 \%$ was reported.

Our approach is to use $\mathrm{SiO}_{2}$ and $\mathrm{Si}_{3} \mathrm{~N}_{4}$ as materials for the lower cladding region of the waveguide. Recently we experimentally demonstrated silicon strip waveguides for $\mathrm{CO}_{2}$ sensing using two different platforms, i.e., silicon strip waveguides on a Si3N4 membrane [4] and on a solid $\left(\mathrm{Si}_{3} \mathrm{~N}_{4}\right) / \mathrm{SiO}_{2}$ substructure [5]. In order to further improve the sensing performance of the waveguide structure, we aim to develop $\mathrm{Si}$ slot waveguides on $\mathrm{SiO}_{2}$ with a significantly higher evanescent field ratio compared to the strip waveguides that were used in our previous work.

Figure 1 shows a schematic representation of the slot waveguide (a) and a representative field distribution of a quasi-TE mode (b), showing strong confinement of the electric field in the slot. The investigated sensor structures are compatible to fabrication on SOI wafers or on standard silicon substrates using deposited $\mathrm{SiO}_{2}$ as substructure materials and deposited poly-silicon as waveguide material. Our ultimate goal is to develop a micro-sensor fully integrated on standard silicon substrates. Therefore, the waveguides were optimized using material parameters that were measured on deposited thin films of poly-Si and $\mathrm{SiO}_{2}$. The waveguides were optimized for a 
wavelength of $\lambda=4.26 \mu \mathrm{m}$, which is at the center of the mid-infrared absorption band of $\mathrm{CO}_{2}$ and for a gap size of $300 \mathrm{~nm}$, which is compatible withstandard micro fabrication processes.
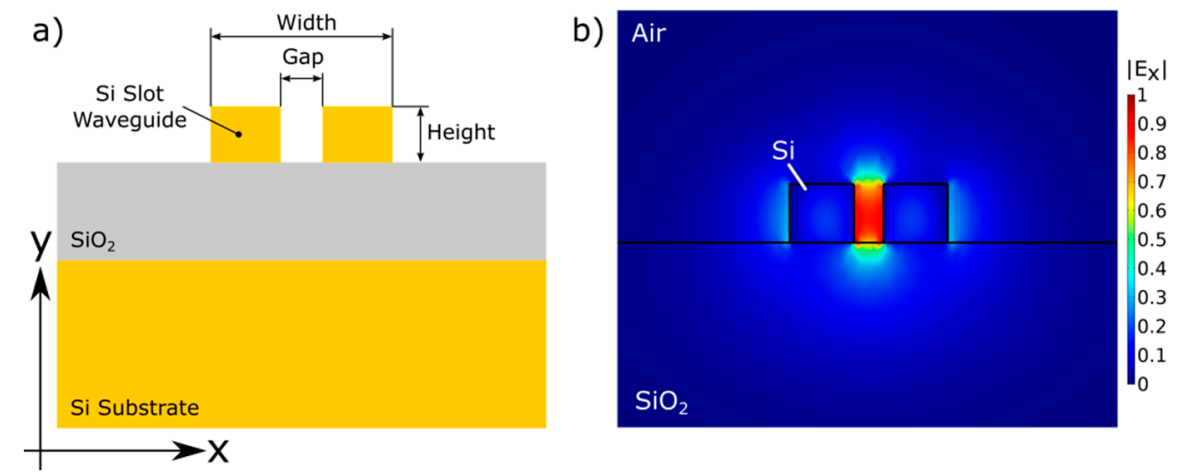

Figure 1. (a) Scheme of the slot waveguide (b) Simulation result depicting the field distribution of $|\mathrm{Ex}|$ for the quasi-TE mode. The results show the strong confinement of the mode in the slot.

\section{Method}

The FEM simulations were conducted using COMSOL Multiphysics 5.3a including the Wave Optics Module. The slot waveguides were investigated using full-vectorial FEM simulations. The domain size was $10 \mu \mathrm{m}$ in $x$ and $y$ direction and was enclosed by perfectly matched layers and scattering boundary conditions in order minimize spurious reflections at the boundaries. So called "Numerical Ports" were applied on both ends of the waveguide which allow to calculate, excite and absorb specific modes. The cross-section of model was meshed with triangular elements with a maximum size of $\lambda /\left(8^{*} n\right)$, where $\lambda$ is the wavelength and $n$ the refractive index of the individual material. Along the propagation direction the mesh of the cross-section was swept with a maximum element size of $\lambda /\left(8^{*} \mathrm{~ns}\right)$. The required optical parameters (i.e., the complex refractive index) of the used materials $\left(\mathrm{SiO}_{2}\right.$ and poly-Si) were measured on thin films prepared by low pressure chemical vapor deposition. The optical parameters were measured using a J.A. Woollam IR VASE ellipsometer.

\section{Results and Discussion}

The real part of the measured refractive index for poly-Si and $\mathrm{SiO}_{2}$ is plotted in Figure 2. While the experimental results were in very good agreement with literature for $\mathrm{SiO}_{2}$ [6], poly-Si had a higher refractive index than previously reported for bulk silicon [7]. As described, the waveguides were designed for a wavelength of $4.26 \mu \mathrm{m}$. The measured real part of the refractive indices at this wavelength are $\mathrm{nsi}_{\mathrm{i}}=3.60$ and $\mathrm{nsio}_{\mathrm{i}}=1.38$. The imaginary part (also known as extinction coefficient) at this wavelength is virtually zero for silicon and was at the noise level of the ellipsometry measurement of $\mathrm{SiO}_{2}$. Therefore, the extinction coefficients were not considered, meaning that losses caused by absorption in the material were not considered in the simulation. 


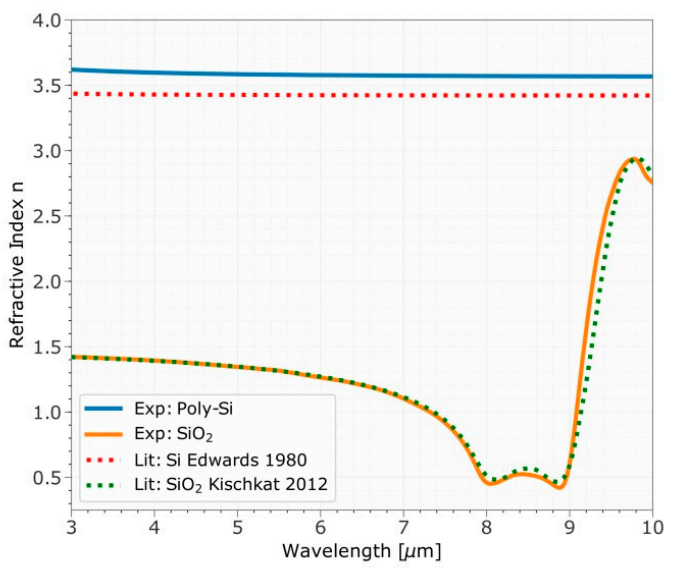

Figure 2. Experimental characterization of the real part of the refractive index $\mathrm{n}$ of the used materials in the mid-infrared region compared to literature values $[6,7]$.

A 3D model of the slot waveguide was developed using COMSOL Multiphysics. The effective mode indices of the fundamental quasi-TE mode were determined conducting a modal analysis on the cross-section of the waveguide (see Figure 3a), searching for the two lowest order modes of the waveguide (modes with the highest effective mode indices). The waveguides were investigated for a gap width of $300 \mathrm{~nm}$. It was found that for geometries with high aspect ratios (height/width) the fundamental mode of the waveguide is the quasi-TM mode, i.e., the mode with the highest effective mode index (not plotted). Furthermore, modes for some geometries with small waveguide widths do not fulfill the condition for guided modes ( $n_{\text {waveguide }}>n_{\text {eff }}>n_{\text {cladding }}$ ) [8]. These modes, i.e., modes with an effective index smaller than the refractive index of $\mathrm{SiO}_{2}$, will leak out and do not propagate over wide ranges in the slot waveguide. Figure $3 c$ ' 1 ' shows a field distribution of such a mode. It is visible that a significant part of the mode is present in the $\mathrm{SiO}_{2}$. The evanescent field ratio (EFR), which is a crucial parameter for the sensing application, was determined using the following equation [3]

$$
E F R=\frac{\iint_{G a s} \vec{S} \cdot \vec{n} d x d y}{\iint_{A l l} \vec{S} \cdot \vec{n} d x d y}
$$

where the surface integrals extend over (parts of) the cross-sectional $x y$-plane of the waveguide, $\vec{S}$ denotes the Poynting vector and $\vec{n}$ the unit normal vector pointing in the direction of propagation. The results are shown in Figure $3 \mathrm{~b}$. The highest EFR among the tested configurations was $42 \%$ for a waveguide height of $800 \mathrm{~nm}$ and a width of $1500 \mathrm{~nm}$ (see also the field distribution plotted in Figure $3 c$ ' 2 '). If the width of the waveguide is further increased, the fraction of the mode that is confined in the silicon part increases, which decreases the EFR of the mode (see Figure $3 \mathrm{~b}$ and also Figure $3 c$ ' 3 '). As indicated in Figure $3 b$, choosing a higher aspect ratio leads to a higher EFR. Nevertheless, a high aspect ratio also favors the propagation of quasi-TM modes. Already for a Si height of $900 \mathrm{~nm}$, the fundamental quasi-TE mode is no longer among two lowest order modes (not plotted). 
a)

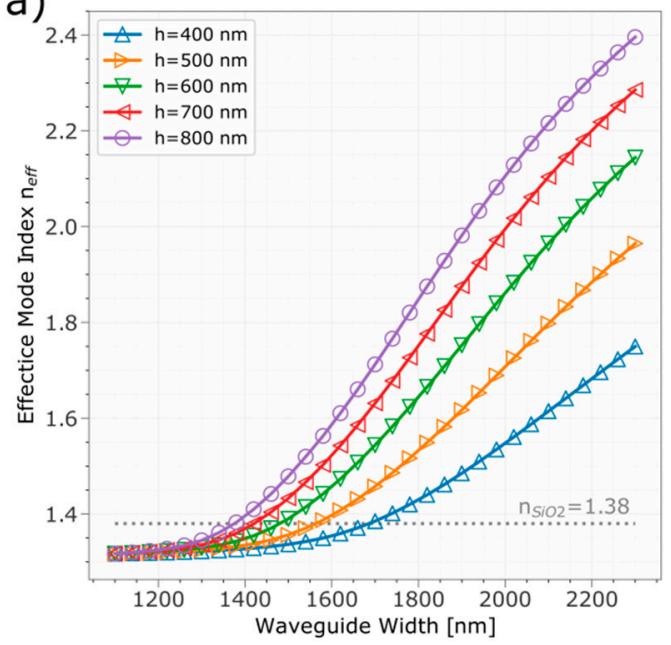

b)

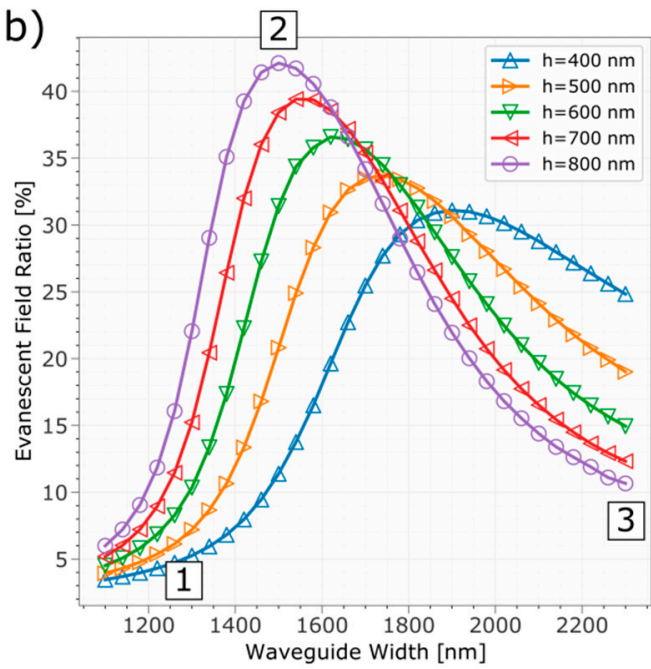

c)
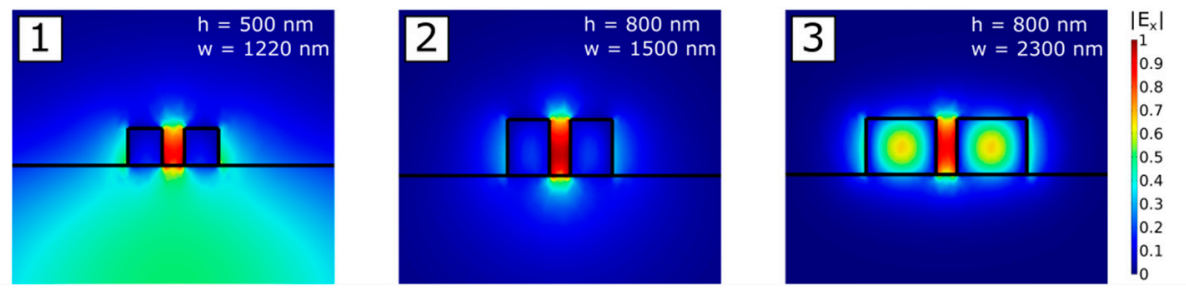

Figure 3. (a) Effective mode index of the fundamental quasi-TE. Modes with effective indices smaller than the effective index of $\mathrm{SiO}_{2}$ (dotted line) are considered unguided. (b) The evanescent field ratio of the investigated waveguides dimensions, depicting the dominant coordinate $|\mathrm{Ex}|$ for the quasi-TE mode. The highest EFR was observed for a height of $800 \mathrm{~nm}$ and a width of $1500 \mathrm{~nm}$. (c) Field distributions of $\mid$ Ex| for some modes.

\section{Conclusions}

A numerical study on slot waveguides for gas sensing, using a $\mathrm{Si}$ on $\mathrm{SiO}_{2}$ platform, was presented. The evanescent field ratio, which is a crucial parameter for the application of waveguides as absorption sensor, was determined. The highest evanescent field ratio that was obtained was $42 \%$. In conclusion, this study indicates that silicon slot waveguides on $\mathrm{SiO}_{2}$ are promising candidates for integrated evanescent field gassensors.

Acknowledgments: This work was performed within the Competence Centre 'ASSIC Austrian Smart Systems Integration Research Center' and 'LCM Linz Centre of Mechatronics', co-funded by the Federal Ministries of Transport, Innovation and Technology (BMVIT) and Digital and Economic Affairs (BMDW) and the Federal Provinces of Carinthia, Styria and Upper Austria within the COMET-Competence Centers for Excellent Technologies Programme. The authors would like to thank Markus Bergmeister for the support with the thin film samples and furthermore Kurt Hingerl and Reza Sharif for the support with the ellipsometry measurements.

Conflicts of Interest: The authors declare no conflict of interest. The founding sponsors had no role in the design of the study; in the collection, analyses, or interpretation of data; in the writing of the manuscript, and in the decision to publish the results.

\section{References}

1. Almeida, V.R.; Xu, Q.; Barrios, C.A.; Lipson, M. Guiding and confining light in void nanostructure. Opt. Lett. 2004, 29, 1209, doi:10.1364/OL.29.001209.

2. $\quad \mathrm{Xu}, \mathrm{Q}$;; Almeida, V.R.; Panepucci, R.R.; Lipson, M. Experimental demonstration of guiding and confining light in nanometer-size low-refractive-index material. Opt. Lett. 2004, 29, 1626, doi:10.1364/OL.29.001626.

3. Huang, Y.; Kalyoncu, S.K.; Zhao, Q.; Torun, R.; Boyraz, O. Silicon-on-sapphire waveguides design for mid-IR evanescent field absorption gas sensors. Opt. Commun. 2014, 313, 186-194, doi:10.1016/j.optcom.2013.10.022. 
4. Ranacher, C.; Consani, C.; Tortschanoff, A.; Jannesari, R.; Bergmeister, M.; Grille, T.; Jakoby, B. Mid-infrared absorption gas sensing using a silicon strip waveguide. Sens. Actuators A Phys. 2018, 277, 117-123, doi:10.1016/j.sna.2018.05.013.

5. Ranacher, C.; Consani, C.; Vollert, N.; Tortschanoff, A.; Bergmeister, M.; Grille, T.; Jakoby, B. Characterization of Evanescent Field Gas Sensor Structures Based on Silicon Photonics. IEEE Photonics Journal. 2018, 10(5):1-4, doi:10.1109/JPHOT.2018.2866628

6. Kischkat, J.; Peters, S.; Gruska, B.; Semtsiv, M.; Chashnikova, M.; Klinkmüller, M.; Fedosenko, O.; Machulik, S.; Aleksandrova, A.; Monastyrskyi, G.; et al. Mid-infrared optical properties of thin films of aluminum oxide, titanium dioxide, silicon dioxide, aluminum nitride, and silicon nitride. Appl. Opt. 2012, 51, 6789, doi:10.1364/AO.51.006789.

7. Edwards, D.F.; Ochoa, E. Infrared refractive index of silicon. Appl. Opt. 1980, 19, 4130, doi:10.1364/AO.19.004130.

8. Ghatak, A.; Thyagarajan, K. An Introduction to Fiber Optics; Cambridge University Press: Cambridge, UK, 1998; ISBN 978-0-521-57785-4.

(C) 2018 by the authors. Licensee MDPI, Basel, Switzerland. This article is an open access article distributed under the terms and conditions of the Creative Commons Attribution (CC BY) license (http://creativecommons.org/licenses/by/4.0/). 